\title{
Mild COVID-19 despite autoantibodies against type I IFNs in autoimmune polyendocrine syndrome type 1
}

\author{
Christian Meisel, ${ }^{1,2}$ Bengisu Akbil, ${ }^{3,4}$ Tim Meyer, ${ }^{1}$ Erwin Lankes, ${ }^{5}$ Victor M. Corman,,${ }^{3,4}$ Olga Staudacher, ${ }^{1,46,7}$ Nadine Unterwalder, ${ }^{1}$ \\ Uwe Kölsch, ${ }^{1}$ Christian Drosten, ${ }^{3,4}$ Marcus A. Mall, ${ }^{4,6,8}$ Tilmann Kallinich, ${ }^{4,6,9}$ Dirk Schnabel, ${ }^{5}$ Christine Goffinet, ${ }^{3,4}$ \\ and Horst von Bernuth ${ }^{1,4,6,7}$
}

\begin{abstract}
'Department of Immunology, Labor Berlin GmbH, Berlin, Germany. ${ }^{2}$ Institute of Medical Immunology, Charité - Universitätsmedizin Berlin, corporate member of Freie Universität Berlin and HumboldtUniversität zu Berlin, Berlin, Germany. ${ }^{3}$ Institute of Virology, and German Centre for Infection Research (DZIF), associated partner, Charité - Universitätsmedizin Berlin, corporate member of Freie Universität Berlin and Humboldt-Universität zu Berlin, Berlin, Germany. ${ }^{4}$ Berlin Institute of Health at Charité - Universitätsmedizin Berlin, Cermany. ${ }^{5}$ Berlin Institute of Health, Center for Chronically Sick Children, Pediatric Endocrinology, Charité - Universitätsmedizin Berlin, corporate member of Freie Universität Berlin, Humboldt-Universität zu Berlin, Berlin, Germany. ${ }^{6}$ Department of Pediatric Respiratory Medicine, Immunology and Critical Care Medicine, Charité - Universitätsmedizin Berlin, corporate member of Freie Universität Berlin, Humboldt-Universität zu Berlin, and Berlin Institute of Health, Berlin, Germany. ${ }^{7}$ Berlin-Brandenburg Center for Regenerative Therapies (BCRT), Charité - Universitätsmedizin Berlin, corporate member of Freie Universität Berlin, Humboldt-Universität zu Berlin, and Berlin Institute of Health (BIH), Berlin, Germany. ${ }^{8}$ Cerman Center for Lung Research (DZL), associated partner, Berlin, Germany. ${ }^{9}$ Deutsches Rheuma-Forschungszentrum (DRFZ), an Institute of the Leibniz
\end{abstract} association, Germany.

\begin{abstract}
Autoantibodies against IFN- $\alpha$ and IFN- $\omega$ (type I IFNs) were recently reported as causative for severe COVID-19 in the general population. Autoantibodies against IFN- $\alpha$ and IFN- $\omega$ are present in almost all patients with autoimmune polyendocrine syndrome type 1 (APS-1) caused by biallelic deleterious or heterozygous dominant mutations in AIRE. We therefore hypothesized that autoantibodies against type I IFNs also predispose patients with APS-1 to severe COVID-19. We prospectively studied 6 patients with APS-1 between April 1, 2020 and April 1, 2021. Biobanked pre-COVID-19 sera of APS-1 subjects were tested for neutralizing autoantibodies against IFN- $\alpha$ and IFN- $\omega$. The ability of the patients' sera to block recombinant human IFN- $\alpha$ and IFN- $\omega$ was assessed by assays quantifying phosphorylation of signal transducer and activator of transcription 1 (STAT1) as well as infection-based IFN-neutralization assays. We describe 4 patients with APS-1 and preexisting high titers of neutralizing autoantibodies against IFN- $\alpha$ and IFN- $\omega$ who contracted SARS-CoV-2, yet developed only mild symptoms of COVID-19. None of the patients developed dyspnea, oxygen requirement, or high temperature. All infected patients with APS-1 were females and younger than 26 years of age. Clinical penetrance of neutralizing autoantibodies against type I IFNs for severe COVID-19 is not complete.
\end{abstract}

\section{Introduction}

Mutations in AIRE (gene encoding the protein autoimmune regulator) cause autoimmune polyendocrine syndrome type 1 (APS-1) (1-3). AIRE is expressed in thymic epithelium and secondary lymphoid organs (4). AIRE regulates promiscuous gene expression of tissue-specific self-antigens in the thymus, a prerequisite for central negative selection of autoreactive T cells. Further, AIRE contributes to the generation of naturally occurring, $\mathrm{CD} 4^{+} \mathrm{CD} 25^{+}$ CD127 $7^{\mathrm{lo} /-} \mathrm{FOXP} 3^{+}$regulatory T cells (5). Patients with APS- 1 develop autoimmunity in endocrine and nonendocrine organs, chronic mucocutaneous candidiasis (CMC), and enamel hypoplasia $(6,7)$. Patients with APS-1 produce autoantibodies against the Th17 cytokines, IFN- $\alpha$ and IFN- $\omega$ (type I IFNs) (8). The role of autoantibodies

Authorship note: CM and BA contributed equally to this work. TM and EL contributed equally to this work. CG and HvB contributed equally to this work. Conflict of interest: VMC together with Euroimmun $\mathrm{GmbH}$ holds a patent regarding SARS-CoV-2 diagnostics via antibody testing (patent application number EP 20158626.0 - 1118/3715847 "A method and reagents for the diagnosis of SARS-CoV-2"). Copyright: (ㄷ 2021, American Society for Clinical Investigation. Submitted: April 26, 2021; Accepted: May 25, 2021; Published: July 15, 2021 Reference information: J Clin Invest. 2021;131(14):e150867. https://doi.org/10.1172/JCl150867. against IL-17 for CMC in patients with APS-1 is well defined (9). In contrast, a role of autoantibodies against type I IFNs for infectious diseases has only recently been suspected, as patients with APS-1 developed severe coronavirus disease 2019 (COVID-19) caused by infection with severe acute respiratory syndrome coronavirus 2 (SARS-CoV-2) (10-12). However, to date there has been no prospective follow-up of patients with APS-1 who contracted SARS-CoV-2.

By blocking a cytokine's biological function, patients with neutralizing anti-cytokine autoantibodies may present with a clinical phenotype resembling corresponding genetic disorders (13). Autoantibodies against type I IFNs were reported in patients with severe COVID-19 (11), among whom a strong bias toward males (95\%) and patients older than 65 years ( $>50 \%)$ was also noted (11). Autoantibodies against type I IFNs in severe COVID-19 were confirmed in additional cohorts (14-17). However, to date, only cohorts collected for severe COVID-19 have been analyzed (11, 15-18). We are not aware of a prospective follow-up of patients with preexisting autoantibodies against type I IFNs. Even if preexisting autoantibodies against type I IFNs are a strong risk factor for severe COVID-19 in preselected cohorts, the clinical penetrance of preexisting neutralizing autoantibodies against type I IFNs for severe COVID-19 is unknown on the individual level as well as on the population level. 


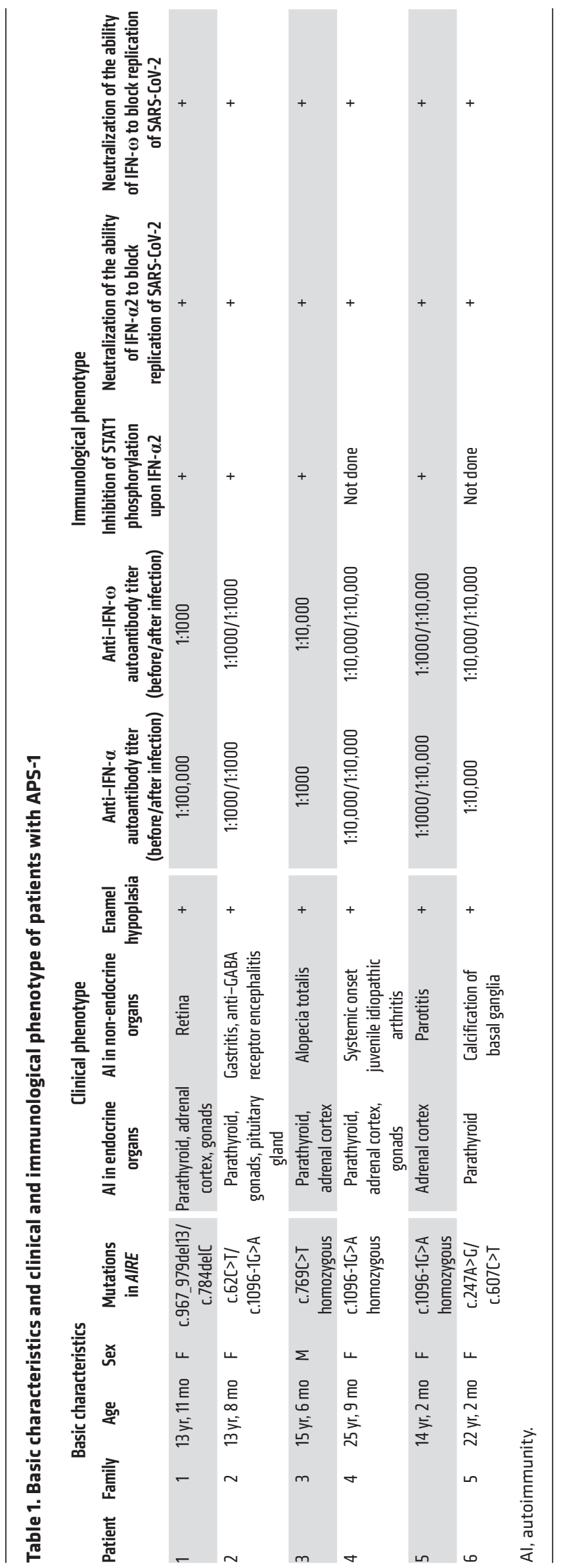

As greater than $95 \%$ of patients with APS-1 develop high titers of neutralizing autoantibodies against type I IFNs (8), APS-1 is a model disease to prospectively study the role of preexisting autoantibodies against type I IFNs for severe COVID-19. To date, 3 patients with APS-1 and severe COIVD-19 (10, 12, 19), as well as severe COVID-19 in 15 of 22 patients in a series of APS-1 patients, have been described (18). We therefore hypothesized that autoantibodies against type I IFNs predispose patients with APS-1 to severe COVID-19. Here, we report on 6 patients with APS-1 and high titers of preexisting neutralizing autoantibodies against IFN- $\alpha$ and IFN- $\omega$, of whom 4 contracted SARS-CoV-2, yet developed mild COVID-19. Our study consists of only patients in regular follow-up for APS-1 who were not recruited due to COVID-19.

\section{Results and Discussion}

Patients with APS-1 develop autoimmunity. Prior to the COVID-19 pandemic, all patients had been followed up at Charité-Universitätsmedizin Berlin for more than 70 patient years (Table 1). Patient 1 is a 13-year-old girl of European descent who developed hypoparathyroidism at 1 year and 4 months of age and adrenal insufficiency at 4 years of age. Compound heterozygous mutations in AIRE were diagnosed. She further developed CMC, retinal degeneration with optical atrophy, and hypergonadotropic hypogonadism. She is treated with hydrocortisone, fludrocortisone, recombinant parathyroid hormone ( $\mathrm{rPTH})$, calcium, magnesium, and sex hormone substitution. She irregularly takes liposomal amphotericin B. Patient 2 is a 13-year-old girl of Arabic origin who presented with hypoparathyroidism at 2 years of age. She experienced an enteroviral meningoencephalitis at 3 years, followed by autoimmune encephalitis at 7 years of age (20). Upon encephalitis, she was treated with plasmapheresis and received mycophenolate mofetil for 36 months. Compound heterozygous mutations in AIRE were diagnosed at 11 years of age. She also developed atrophic gastritis, growth hormone deficiency, and hypergonadotropic hypogonadism. She is treated with $\mathrm{rPTH}$, calcium, vitamin $\mathrm{D}$, and recombinant human growth hormone. Patient 3 is a 15 -year-old boy of European descent who presented with hypoparathyroidism at 8 years of age, when adrenal insufficiency was also noticed and a homozygous mutation in AIRE was identified. At 10 years of age he developed alopecia totalis. He is treated with calcium, calcitriol, hydrocortisone, and fludrocortisone. Patient 4 is a 25 -year-old woman of Arabic origin who had been treated for systemic onset juvenile idiopathic arthritis before being diagnosed with hypoparathyroidism at 11 years and adrenal insufficiency at 13 years of age. The diagnosis of APS-1 became evident at 22 years of age. APS- 1 is most likely caused by the same homozygous mutation in $A I R E$ as in her younger sister (patient 5). Patient 4 is treated with calcitriol, calcium, hydrocortisone, fludrocortisone, and estradiol for ovarian insufficiency. Patient 5, the younger sister of patient 4 , is a 14-year-old girl. At 2.5 years of age she presented with unilateral parotitis and adrenal insufficiency at 8 years of age. A homozygous mutation in AIRE was found at 11 years of age. She is treated with hydrocortisone and fludrocortisone. Patient 6 is a 22-year-old woman of Turkish origin who developed hypoparathyroidism at 4 years of age. Compound heterozygous mutations in AIRE were diagnosed at 4 years of age. She receives calcitriol. All patients show enamel hypoplasia. 
Table 2. Serology for SARS-CoV-2 in patients with APS-1

\begin{tabular}{|c|c|c|c|c|c|c|c|c|c|c|}
\hline \multirow[b]{2}{*}{ Patient } & \multirow[b]{2}{*}{$\begin{array}{l}\text { Time point relative } \\
\text { to SARS-CoV-2 infection }\end{array}$} & \multicolumn{2}{|c|}{ S1-IgG ELISA } & \multicolumn{2}{|c|}{ S1-IgA ELISA } & \multicolumn{5}{|c|}{ SARS-CoV-2 IgC SeraSpot } \\
\hline & & S1-IgC ratio & S1-IgG result & S1-IgA ratio & S1-IgA result & $\mathbf{N}$ & RBD & S1 & Complete spike & Result \\
\hline 1 & No infection reported & 0.1 & Neg & 0.6 & Neg & 0 & 0 & 0 & 0 & Neg \\
\hline 2 & After & 3.89 & Pos & 5.6 & Pos & 0.6 & 2.6 & 1.1 & 1.5 & Pos \\
\hline 3 & No infection reported & 0.07 & Neg & 0.55 & Neg & 0 & 0 & 0 & 0 & Neg \\
\hline 4 & Before & 0.07 & Neg & 0.54 & Neg & 0 & 0 & 0 & 0 & Neg \\
\hline 5 & Before & 0.09 & Neg & 0.36 & Neg & 0.1 & 0 & 0.1 & 0 & Neg \\
\hline 5 & After & 8.24 & Pos & $>12$ & Pos & 3.1 & 5.9 & 4.6 & 5.1 & Pos \\
\hline 6 & Before & 0.06 & Neg & 0.37 & Neg & 0 & 0 & 0 & 0 & Neg \\
\hline 6 & After & 3.08 & Pos & 1.7 & Pos & 0.3 & 2.0 & 0.6 & 1.0 & Pos \\
\hline
\end{tabular}

S1, subunit S1 of spike protein; N, nucleocapsid; RBD, S1 receptor-binding domain. S1 ratios were calculated by dividing the measured optical density of the specific serum by that of a cutoff control tested in parallel on each ELISA plate.

Infections with SARS-CoV-2 caused mild COVID-19 in 4 patients with APS-1. Patient 2 presented with vomiting, headache, and rhinitis. SARS-CoV-2 smear was positive. Three days later, smell and taste sense were absent. Fatigue, temperatures up to $38.5^{\circ} \mathrm{C}$, slight pain in both knees, as well as headaches for 10 days were reported. Smell and taste returned 10 days after onset of symptoms. Patient 4 presented with up to $39^{\circ} \mathrm{C}$, flu-like symptoms, and cough. SARS-CoV-2 smear was positive. Symptoms resolved after 7 days. Patient 5, living in the same household as patient 4, reported mild rhinitis, cough for 5 days, and normal body temperature. In patient 6 , SARS-CoV-2 was suspected because of a positive test in the household. The patient reported cough, rhinitis, headaches, myalgia, a sore throat, normal body temperature, and loss of taste for 4 days. After 7 days all symptoms resolved apart from fatigue for 1 more week. As patients developed neither high fever nor dyspnea, all were seen by their local physician and adhered to quarantine measures. None of the patients was admitted to the hospital. When quarantine measures were lifted, serology for SARS-CoV-2 was performed. All patients who reported SARS-CoV-2 infectioncompatible symptoms proved seropositive for antibodies specific for SARS-CoV-2 (Table 2). In summary, 4 patients with APS-1 contracted SARS-CoV-2 but all presented with mild COVID-19.

Patients with APS-1 have high titers of preexisting neutralizing autoantibodies against type I IFNs. We assessed preexisting sera of all APS- 1 patients for autoantibodies against IFN- $\alpha$, IFN- $\omega$, IFN- $\beta$, IL-6, IFN- $\gamma$, and GM-CSF. All were positive for autoantibodies against IFN- $\alpha$ and IFN- $\omega$, none for autoantibodies against IFN- $\beta$, IL-6, IFN- $\gamma$, or GM-CSF (Figure 1A). Dilution experiments showed high titers of autoantibodies against IFN- $\alpha$ and IFN- $\omega$, as a serum dilution of up to 1:100,000 was necessary to reach background levels of healthy, autoantibody-negative controls (Figure 1, B and C). Titers of autoantibodies against type I IFNs rose slightly in APS-1 patients upon infection with SARS-CoV-2 (Supplemental Figure 1; supplemental material available online with this article; https://doi.org/10.1172/JCI150867DS1). Neutralizing activity of autoantibodies against IFN- $\alpha$ was assessed by comparing STAT1 phosphorylation in monocytes upon ex vivo stimulation with recombinant IFN- $\alpha 2$ in whole blood of a healthy control and in patients. Although $1 \mathrm{ng} / \mathrm{mL}$ IFN- $\alpha 2$ was sufficient to induce maximum STAT1 phosphorylation in monocytes in whole blood from a healthy donor, the phospho-STAT1 signal in samples from APS-1 patients was suppressed even after stimulation with $10 \mathrm{ng} / \mathrm{mL}$ IFN- $\alpha 2$. In contrast, IFN- $\gamma$-induced STAT1 phosphorylation was similar between patients and the control sample (Figure 1D).

Type I IFN-mediated inhibition of SARS-CoV-2 replication is abolished by autoantibodies in patients' plasma in vitro. Neutralizing activity of autoantibodies against IFN- $\alpha$ and IFN- $\omega$ was further assessed by quantifying their ability to nullify the antiviral effect of exogenous IFN in a SARS-CoV-2 infection model of respiratory epithelial Calu-3 cells. As expected, treatment of cells with recombinant IFN- $\alpha 2 \mathrm{a}$ and IFN- $\omega$ in the absence of serum or in the presence of a healthy individual's serum reduced their susceptibility to SARS-CoV-2 infection, as assessed by quantification of viral RNA in culture supernatant (Figure 2, A and B). In contrast, SARSCoV-2 efficiently infected Calu-3 cells that were inoculated with the patients' sera, even in the presence of fixed doses of IFN- $\alpha 2 \mathrm{a}$ (Figure 2A) and IFN- $\omega$ (Figure 2B), respectively. In general, IFN neutralization was serum concentration dependent. Specifically, for most sera, virus replication in the presence of a fixed dose of type I IFN was strongest when Calu-3 cells were incubated with $1 \%$ patient sera and weakest when incubated with $0.001 \%$ patient sera (Supplemental Figure 2, A and B). Interestingly, we failed to out-titrate the serum of patient 1 in the presence of IFN- $\alpha 2 a$, indicating high anti-IFN- $\alpha 2$ a neutralization capacity, which is in line with the highest titer of autoantibodies in this serum (Figure 1). The neutralizing activity of autoantibodies against IFN was further confirmed by assessing the infectivity of released virions (Figure 2, D-F, and Supplemental Figure 3). In the absence of IFNs and serum, inoculation of cells with SARS-CoV-2 gave rise to abundant de novo virus production. Addition of exogenous IFNs efficiently prevented virus production, both in the absence of serum and in the presence of serum, of an autoantibody-negative individual. However, incubation of cells with individual patient sera allowed efficient production of infectious virions even in the presence of IFN- $\alpha 2$ a (Figure 2D) and IFN- $\omega$ (Figure 2E), confirming efficient neutralization of antiviral IFNs, mirroring our results obtained by 
A

Anti-cytokine autoantibodies
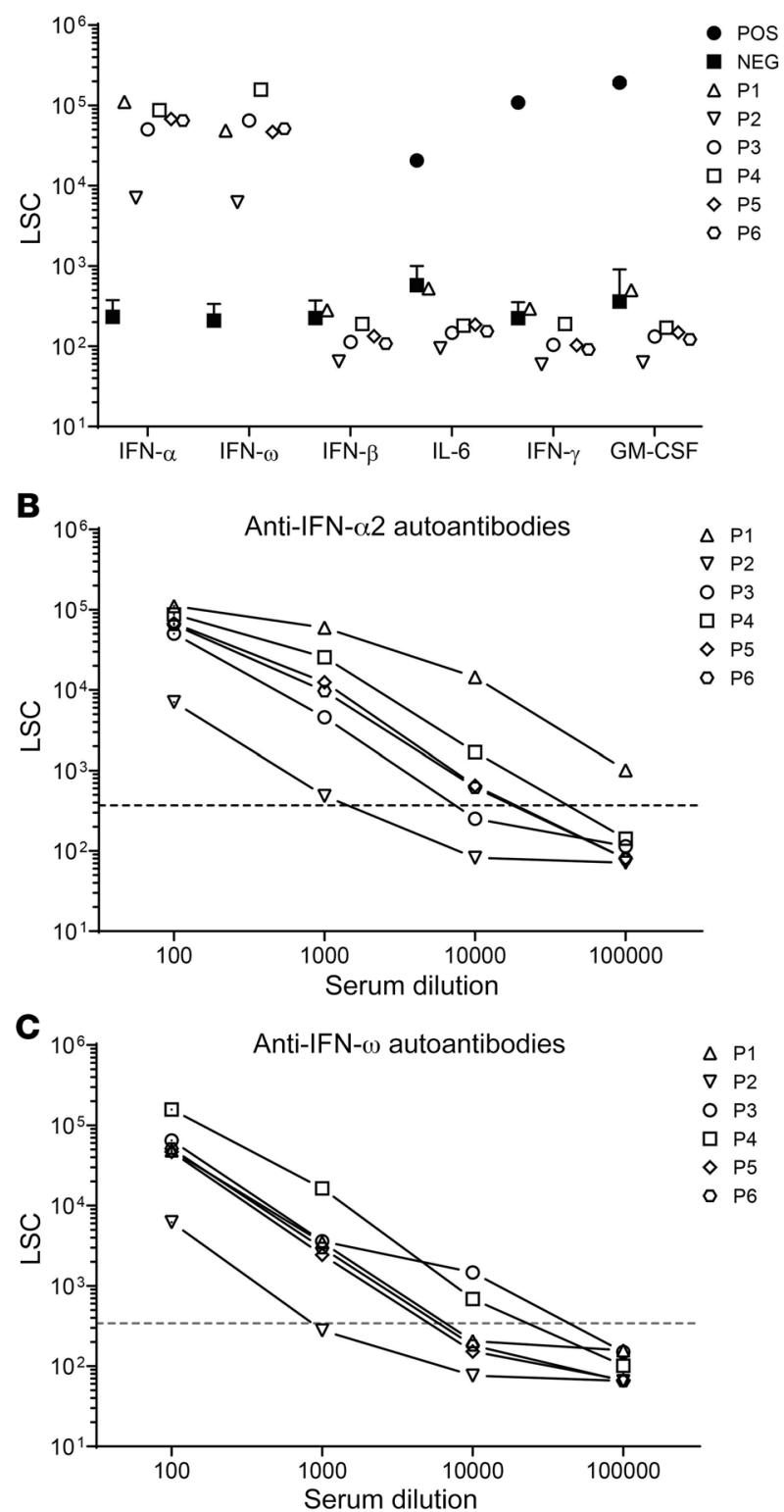

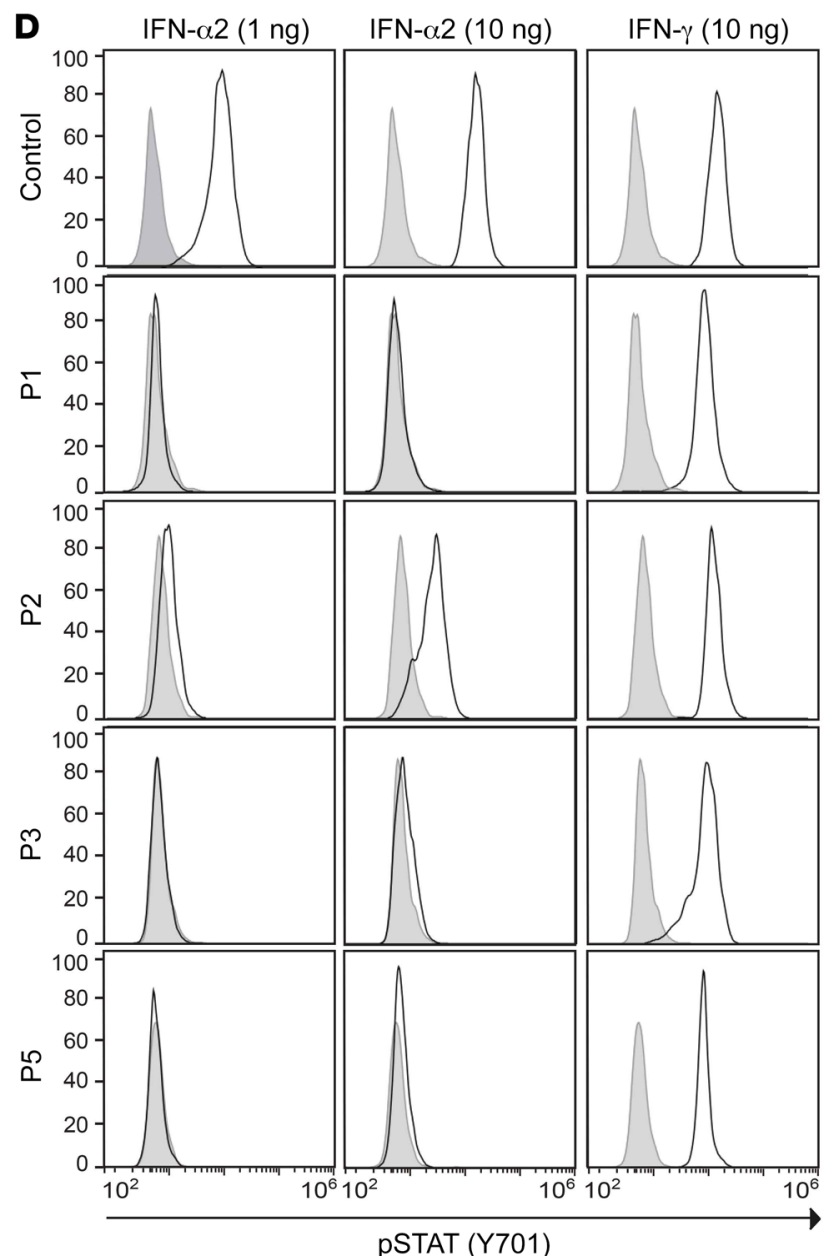

Figure 1. Neutralizing auto-Abs against IFN- $\alpha 2$ and IFN- $\omega$ in patients with APS-1. (A) Detection of IgG auto-Abs against IFN- $\alpha 2$, IFN- $\omega$, IFN- $\beta$, IFN- $\gamma$, IL-6, and CM-CSF in sera (1:100 dilution) from patients with APS-1 (P1-P6), healthy controls (NEG, $n=17$ ), and patients with known auto-Abs against IFN- $\gamma$, IL-6, and GM-CSF (POS, $n=1$ ). Detection of auto-Abs against (B) IFN- $\alpha 2$ and (C) IFN- $\omega$ in serially diluted patient sera. Dotted lines indicate the maximum light signal counts (LSC) in the anti-IFN- $\alpha 2$ and anti-IFN- $\omega$ assay in the cohort of healthy controls. (D) FACS histograms depicting STAT1 phosphorylation (p-STAT1) in whole-blood monocytes from a healthy control and 4 APS-1 patients stimulated with IFN- $\alpha 2$ (1 and $10 \mathrm{ng} / \mathrm{mL})$ or IFN- $\gamma(10 \mathrm{ng} / \mathrm{mL})$.

real-time reverse transcription PCR (qRT-PCR) (Figure 2, A and B). IFN neutralization was generally serum concentration dependent, again with the exception of the serum of patient 1 in the presence of IFN- $\alpha 2 a$ (Supplemental Figure 2, C and D). Importantly, in the absence of IFNs, healthy individuals' and patients' sera did not modulate infection efficiency as compared with the condition without serum addition (Figure 2, C and F), arguing for a specific proviral effect exerted by the patients' sera that manifests itself specifically in the presence of IFNs. In summary, all patients with APS-1 in our cohort exhibited autoantibodies at titers that are sufficient for functional neutralization of type I IFNs in an IFN-sensitive SARS-CoV-2 infection assay.
Mild COVID-19 despite high titers of neutralizing autoantibodies against type I IFNs in 4 patients with APS-1. Here, we describe 4 patients with APS-1 and high titers of preexisting, neutralizing autoantibodies against type I IFNs who experienced only mild COVID-19. Our observation may seem difficult to reconcile with reports of 3 patients with APS-1 who developed severe COVID-19 $(10,12,19)$. Further, autoantibodies against type I IFNs were described as a risk factor for severe COVID-19 in at least 10\% of patients with severe COVID-19 (11). Recently, a study described severe COVID-19 in 15 of 22 patients in a cross-sectional case series of patients with APS-1; however, 7 patients of the same cohort of 22 developed mild to moderate COVID-19, of whom 3 were not even 

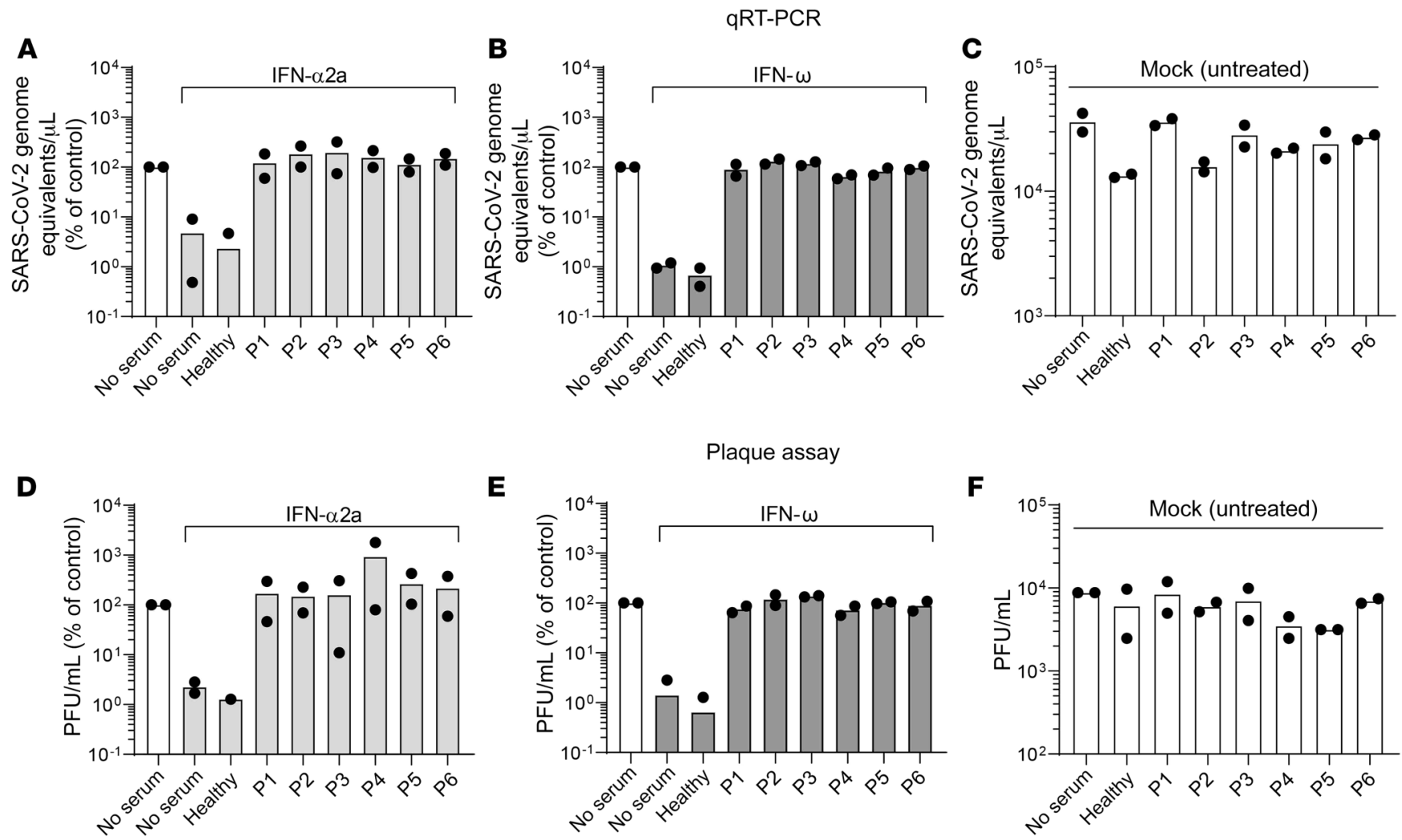

Figure 2. Auto-Abs in patients with APS-1 neutralize the ability of type I IFNs to inhibit SARS-CoV-2 infection. Calu-3 cells were mock treated (no serum) or pretreated with indicated concentrations of human serum in the presence or absence of $200 \mathrm{IU} / \mathrm{mL}$ IFN- $\alpha 2 \mathrm{a}$ (A and D) or $5 \mathrm{ng} / \mathrm{mL}$ IFN- $\omega$ (B and E) for 16 hours before infection. IFN and serum were removed, and cells were infected with SARS-CoV-2 at an MOI of 0.01 for 1 hour, washed, and fresh medium was applied to the cells. Twenty-four hours after infection, supernatant was harvested for viral RNA extraction and plaque assays. (A-C) Viral RNA was extracted from supernatant and SARS-CoV-2 genome equivalents/ $\mu \mathrm{L}$ were quantified by qRT-PCR using primers targeting the E gene region. (D-F) Supernatants were titrated on Vero E6 cells and incubated for plaque formation for 3 days. Plaques were counted and PFU/mL was determined. Data were generated in 2 independent assays. Values obtained in the absence of serum and IFN were set to $100 \%$.

hospitalized (18). SARS-CoV-2 is sensitive to the antiviral properties of type I IFNs, as has been shown extensively in vitro, ex vivo, and in vivo (21). Therefore, it appears intuitive that interference with these cytokines results in a worsened outcome of SARS-CoV-2 infection. Strikingly, all individuals with high titers of preexisting and neutralizing autoantibodies against type I IFNs yet mild COVID-19 in our study were young females $(13,14,22$, and 25 years of age), whereas a pronounced excess of males older than 65 years was noted among most patients with autoantibodies against type I IFNs and severe COVID-19 (11). We were not able to verify to what extent autoantibodies against IFN- $\alpha$ and IFN- $\omega$ block the respective IFNs in our patients in vivo. So, our surprising observation of mild COVID-19 despite high titers of neutralizing autoantibodies against both IFN- $\alpha$ and IFN- $\omega$ in young females may be explained by the assumption that these autoantibodies do not fully neutralize either type I IFN in vivo. Consequently, if autoantibodies against IFN- $\alpha$ and IFN- $\omega$ do not completely block, but only dampen the biological activity of, IFN- $\alpha$ and IFN- $\omega$ in vivo, older males may exhibit additional risk factors for severe COVID-19 that are yet absent or less frequent/ less present in most young patients and/or females.

Rescue treatment in patients with APS-1 only in severe COVID-19. In conclusion, even if preexisting autoantibodies against type I IFNs increase the risk for severe COVID-19, penetrance for severe
COVID-19 is not complete. Importantly, and in contrast to previous studies $(10,12,18,19)$, our report is the first to our knowledge based on a prospective follow-up of patients with preexisting autoantibodies against type I IFNs. Large prospective studies may help to estimate the true risk of patients with preexisting autoantibodies against type I IFNs, such as in patients with APS-1 for severe COVID-19. As clinical penetrance for severe COVID-19 in the presence of preexisting autoantibodies against type I IFNs is unclear at the population and individual level, we do not advise admitting all patients with APS- 1 who contracted SARS-CoV- 2 to the hospital for upfront therapies (e.g., monoclonal antibodies, IFN- $\beta$, plasmapheresis). Nevertheless, we strongly advise informing all patients with autoantibodies against type I IFNs about their increased risk for severe COVID-19. As severe COVID-19 has been described also in young and in female patients with APS-1, all patients with APS-1 who contracted SARS-CoV-2 must be followed up closely.

\section{Methods}

A complete description of the materials and methods can be found in the supplemental material.

Study approval. All procedures performed in studies involving human participants were in accordance with the ethical standards of the institutional and/or national research committee (Charité 
- Universitätsmedizin Berlin, Germany, EA2/132/11) and with the 1964 Helsinki declaration and its later amendments or comparable ethical standards. Written informed consent was obtained from all individual participants included in the study.

\section{Author contributions}

CM, CG, and HVB planned the study. TM and OS assessed autoantibodies and STAT1 phosphorylation. BA assessed IFN neutralization in Calu-3 cells. TK, EL, and DS recruited patients. CD provided the SARS-CoV-2 isolate. VMC generated serology data. MAM, UK, and NU critically discussed the manuscript. HVB wrote the initial version of the manuscript, and CM, CG, and HVB completed the final version. All authors read and approved the final version of the manuscript.

\section{Acknowledgments}

We thank the patients and their families for ongoing trust and collaboration. We thank Mahtab Maleki and Jenny Jansen for excellent technical support. This work was supported by the Innovationsfond of Labor Berlin (to CM, CG, and HVB); by funding from the Deutsche Forschungsgemeinschaft (DFG) Collaborative Research Centre CRC900 "Microbial Persistence and its Control," project number 158989968, project C8 (awarded to CG); by funding from the Berlin Institute of Health (BIH) (to CG); and by the German Federal Ministry for Education and Research (82DZL0098B1 and 01IK20337 to MAM). We thank Jean-Laurent Casanova for discussions on our manuscript.

Address correspondence to: Christine Goffinet, Institute of Virology, Charité - Universitätsmedizin Berlin, Charitéplatz 1, D-10117 Berlin, Germany. Phone: 49.30.450.525489; Email: christine. goffinet@charite.de. Or to: Horst von Bernuth, Department of Pediatric Respiratory Medicine, Immunology and Critical Care Medicine, Charité - Universitätsmedizin Berlin, Augustenburger Platz 1, D-13353 Berlin, Germany. Phone: 49.30.450.566693; Email: horst.von-bernuth@charite.de.
1. Nagamine K, et al. Positional cloning of the APECED gene. Nat Genet. 1997;17(4):393-398.

2. Finnish-German APECED Consortium. An autoimmune disease, APECED, caused by mutations in a novel gene featuring two PHD-type zincfinger domains. Nat Genet. 1997;17(4):399-403.

3. Oftedal BE, et al. Dominant mutations in the autoimmune regulator AIRE are associated with common organ-specific autoimmune diseases. Immunity. 2015;42(6):1185-1196.

4. Wang HX, et al. Thymic epithelial cells contribute to thymopoiesis and T cell development. Front Immunol. 2019;10:3099.

5. Perniola R. Twenty years of AIRE. Front Immunol. 2018;9:98.

6. Ahonen P, et al. Clinical variation of autoimmune polyendocrinopathy-candidiasis-ectodermal dystrophy (APECED) in a series of 68 patients. N Engl JMed. 1990;322(26):1829-1836.

7. Ferre EM, et al. Redefined clinical features and diagnostic criteria in autoimmune polyendocrinopathy-candidiasis-ectodermal dystrophy. JCI Insight. 2016;1(13):e88782.

8. Meager A, et al. Anti-interferon autoantibodies in autoimmune polyendocrinopathy syndrome type 1. PLoS Med. 2006;3(7):e289.

9. Puel A, et al. Autoantibodies against IL-17A,
IL-17F, and IL-22 in patients with chronic mucocutaneous candidiasis and autoimmune polyendocrine syndrome type I. J Exp Med. 2010;207(2):291-297.

10. Beccuti G, et al. A COVID-19 pneumonia case report of autoimmune polyendocrine syndrome type 1 in Lombardy, Italy: letter to the editor. J Endocrinol Invest. 2020;43(8):1175-1177.

11. Bastard P, et al. Autoantibodies against type I IFNs in patients with life-threatening COVID-19. Science. 2020;370(6515):eabd4585.

12. Lemarquis A, et al. Severe COVID-19 in an APS1 patient with interferon autoantibodies treated with plasmapheresis [published online April 16, 2021]. J Allergy Clin Immunol. https://doi. org/10.1016/j.jaci.2021.03.034.

13. Ku CL, et al. Autoantibodies against cytokines: phenocopies of primary immunodeficiencies? Hum Genet. 2020;139(6-7):783-794.

14. de Prost N, et al. Plasma exchange to rescue patients with autoantibodies against type I interferons and life-threatening COVID-19 pneumonia. J Clin Immunol. 2021;41(3):536-544.

15. van der Wijst MGP, et al. Longitudinal single-cell epitope and RNA-sequencing reveals the immunological impact of type 1 interferon autoantibodies in critical COVID-19 [preprint]. https:// doi.org/10.1101/2021.03.09.434529. Posted on bioRxiv, March 10, 2021.

16. Troya J, et al. Neutralizing autoantibodies to type I IFNs in $>10 \%$ of Patients with severe COVID19 pneumonia hospitalized in Madrid, Spain [published online April 13, 2021]. J Clin Immunol. https://doi.org/10.1007/s10875.021.01036-0.

17. Koning R, et al. Autoantibodies against type I interferons are associated with multi-organ failure in COVID-19 patients [published online April 9 ,2021]. Intensive Care Med. https://doi. org/10.1007/s00134.021.06392-4.

18. Bastard P, et al. Preexisting autoantibodies to type I IFNs underlie critical COVID-19 pneumonia in patients with APS-1. JExp Med. 2021;218(7):e20210554.

19. Carpino A, et al. Autoimmune polyendocrinopathy-candidiasis-ectodermal dystrophy in two siblings: same mutations but very different phenotypes. Genes (Basel). 2021;12(2):169.

20. Nikolaus $M$, et al. Severe GABA receptor encephalitis without seizures: A paediatric case successfully treated with early immunomodulation. Eur J Paediatr Neurol. 2018;22(3):558-562.

21. Schultze JL, Aschenbrenner AC. COVID-19 and the human innate immune system. Cell. 2021;184(7):1671-1692. 